\title{
Evaluación de la calidad de la energía en Radio Universitaria
}

\section{Evaluation of the quality of the energy in Radio Universitaria}

\author{
${ }^{1}$ Cueva Ríos, Percy Humberto; Orellana Mendoza, Wilar Tito \\ Facultad de Ingeniería Eléctrica y Electrónica, Universidad Nacional del Centro del Perú \\ Email: pcueva@uncp.edu.pe
}

\section{Resumen}

El presente trabajo de investigación, tiene por finalidad determinar la calidad de la energía en la estación transmisora de Radio Universitaria. El estudio es de tipo descriptivo-aplicativo que analiza las mediciones eléctricas para determinar que la calidad de la energía esté dentro de los rangos mínimos aceptables en los parámetros eléctricos según la NTCSE. El presente estudio está enmarcado dentro de la línea de investigación sobre calidad del servicio.

Para determinar los parámetros eléctricos y obtener una mejor caracterización de los armónicos con fines de la evaluación con la NTCSE, se tomaron mediciones cada 15 minutos para la tensión RMS, para las armónicas individuales de tensión, THD de tensión y armónicas de corriente; mediante una evaluación y análisis de las variaciones de los parámetros eléctricos se determinó que la calidad de energía de la estación transmisora de Radio Universitaria no es de buena porque los parámetros eléctricos no está dentro de los límites permisibles de la NTCSE, por lo que se recomienda la utilización de filtros de armónicos de 5to orden y 7 mo orden para disminuir los efectos de variación de tensión y corriente.

Palabras Clave: calidad de la energía, armónico de tensión, armónico de corriente.

\begin{abstract}
The purpose of this research is to determine the quality of the energy in the transmitting station of Radio Universitaria. The research type is descriptive-application which analyzes the electrical measurements to determine if the quality of the energy is within the minimum acceptable ranges of electrical parameters according to the NTCSE. This study is framed within the line of research on service quality.

In order to determine the electrical parameters and obtain a better characterization of the harmonics for the purposes of the evaluation with the NTCSE, measurements were taken every 15 minutes for the RMS voltage, for individual voltage harmonics, voltage THD and current harmonics by means of an evaluation and analysis of the variations of the electrical parameters it was determined that the quality of energy of the transmitting station of Radio Universitaria lacks good quality because the electrical parameters are not within the allowable limits of the NTCSE, therefore, the use of 5 th order and 7 th order harmonic filters is recommended to reduce the effects of voltage and current variation.
\end{abstract}

Keywords: power quality, voltage harmonic, current harmonic. 


\section{Introducción}

En la actualidad el estudio de la calidad de la energía eléctrica ha adquirido mucha importancia y, tal vez, la razón más importante es la búsqueda del aumento de productividad y competitividad de las empresas; asimismo, porque existe una interrelación entre calidad de la energía eléctrica, la eficiencia y la productividad.

La calidad de la energía eléctrica puede definirse como una ausencia de interrupciones, sobre tensiones y deformaciones de onda producidas por armónicas en la red y variaciones de voltaje RMS suministrado al usuario; esto referido a la estabilidad del voltaje, la frecuencia y la continuidad del servicio eléctrico. Asimismo, se ha determinado que uno de los problemas más comunes que ocasiona el desperdicio de energía eléctrica en las empresas es la calidad de esta, pues influye en la eficiencia de los equipos eléctricos que usan la energía eléctrica.

Los problemas se presentan no solo con la variación de la tensión. Los sistemas basados en electrónica de potencia, además de ser sensibles a las variaciones de la tensión, también causan perturbaciones que afectan a la red eléctrica asociada al suministro. El problema se agrava con el creciente uso de sistemas de mayor eficiencia, basados en equipos alimentados o accionados mediante convertidores estáticos de potencia que generan perturbaciones como armónicos flickers, etc. que generan errores en las mediciones, operación incorrecta de sistemas de protección y daño en elementos de las instalaciones eléctricas. Las variaciones de la calidad de la energía eléctrica ocasionan fallas que paralizan la producción ocasionando tiempo perdido y costos de producción inesperados. Los errores en las mediciones influyen en la facturación por energía eléctrica; por ello, es importante realizar un estudio de la calidad de la energía para obtener un ahorro en el consumo eléctrico.

El objetivo del presente trabajo es determinar la calidad de la energía en la estación transmisora de Radio Universitaria mediante mediciones eléctricas, para establecer que la calidad de la energía esté dentro de los rangos mínimos aceptables en los parámetros eléctricos según la NTCSE. Además, proponer un filtro de armónicos que podría requerir las instalaciones para mejorar la calidad de energía.

\section{Materiales y métodos}

\section{Materiales y equipos a utilizar}

- Analizador de Redes.

- Computadora con Internet.

- Libros de Consulta

- Revistas

- Memoria USB

- Útiles de Escritorio

\section{Técnicas e instrumentos y fuentes de recolección de datos}

\section{Lugar y periodo de ejecución}

Se realizará en las instalaciones de la estación transmisora de Radio Universitaria de la Universidad Nacional del Centro del Perú.

\section{Población y muestra}

\section{Población}

La población considera todos los valores en el tiempo de los parámetros de tensión y corriente.

\section{Muestra}

Mediciones de los armónicos de tensión y corriente durante 07 días.

\section{Tipo de Investigación}

El trabajo de investigación consiste en realizar la evaluación de la calidad de energía de Radio Universitaria de la Universidad Nacional del Centro del Perú, en el año 2016; por tanto, es una investigación de tipo descriptivo. De acuerdo con Goldhor, citado en el trabajo de investigación de Mauro Coarite Mamani (2007), los estudios descriptivos se concentran en medir con la mayor exactitud posible la variable planteada; por ello el investigador debe definir qué se va a medir y cómo lograr precisión en esa medición.

El presente estudio causal comparativo analiza las mediciones de los armónicos de tensión y corriente. Después de realizar las comparaciones de las mediciones, con los límites permitidos por la NTCSE, se podrá describir los factores que parecen explicar la presencia de una buena o deficiente calidad de energía.

Las mediciones serán programadas en coordinación con el personal a cargo de la supervisión de trabajos. Cabe precisar que los puntos eléctricos evaluados han sido etiquetados a través de un código a fin de ubicar cualquier tipo de curva o evaluación.

El período de medición será de 7 días continuos cumpliendo con lo establecido por la NTCSE para el punto eléctrico. Los parámetros serán registrados a intervalos de un minuto, con la finalidad de obtener una mejor caracterización de los armónicos. Asimismo, para fines de la evaluación con la NTCSE, se evaluarán los valores a 15 minutos para la tensión RMS y 10 minutos para las armónicas individuales y THD de tensión y corriente

\section{Nivel de medición}

Investigación Cuantitativa-Comparativa: Para la investigación cuantitativa se maneja la recolección y el análisis de datos; mediante el uso de la comparación se establece información confiable del comportamiento de los parámetros eléctricos, verificando si estos se encuentran dentro o fuera de los límites establecidos por la NTCSE. 


\section{Diseño de investigación}

La investigación es descriptivo simple: se emplea esté diseño cuando el investigador está interesado en recoger información acerca de una variable en una muestra. Por ejemplo: Si se tiene un problema de investigación como: ¿Cómo determinar la calidad de la energía en la estación transmisora de Radio Universitaria? Se tomó una muestra $(\mathrm{M})$ de las mediciones de los armónicos de tensión y corriente, luego se observa a los comportamientos de estos parámetros, en tanto que se trata de un diseño descriptivo no interviene en la variable, simplemente la observa y describe. De ahí que su esquema es:

$$
\mathrm{M} \longrightarrow \mathrm{Xi} \longrightarrow \mathrm{O}
$$

M: Muestra de elementos o Población de elementos de estudio $(\mathrm{P})$.

Xi: Variable(s) de estudio, calidad de energía.

O: Resultados de la medición de los armónicos de tensión y corriente.

\section{Resultados}

Resultados de las mediciones realizadas en las instalaciones de Radio Universitaria:

\section{Resultados de tensión RMS}

Gráfico $\mathbf{N}^{\circ}$. 01. Comportamiento de la tensión RMS

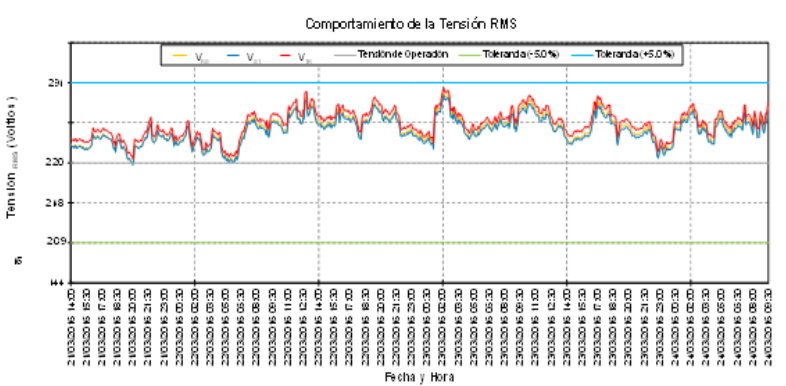

\section{Resultados de las armónicas de tensión}

Tabla 03. Distorsión de armónicos de tensión

\begin{tabular}{|c|c|c|c|c|c|c|c|c|}
\hline \multirow[b]{2}{*}{ Ítem } & \multirow[b]{2}{*}{$\begin{array}{l}\text { Punto } \\
\text { de Me- } \\
\text { dición }\end{array}$} & \multirow{2}{*}{$\begin{array}{c}\text { Ten- } \\
\text { sión } \\
\text { No- } \\
\text { minal } \\
\text { (kV) }\end{array}$} & \multicolumn{2}{|c|}{ Vh - 5 (\%) } & \multicolumn{2}{|c|}{ Vh - 7 (\%) } & \multicolumn{2}{|c|}{$\mathrm{Vh}-11(\%)$} \\
\hline & & & $\begin{array}{l}\text { Valor } \\
\text { medi- }\end{array}$ & & ii- & & & \\
\hline & & 0.22 & 1.50 & 0.00 & 1.14 & 5.00 & 0.81 & 3.5 \\
\hline
\end{tabular}

Gráfico 02. Espectro de la armónica de tensión.

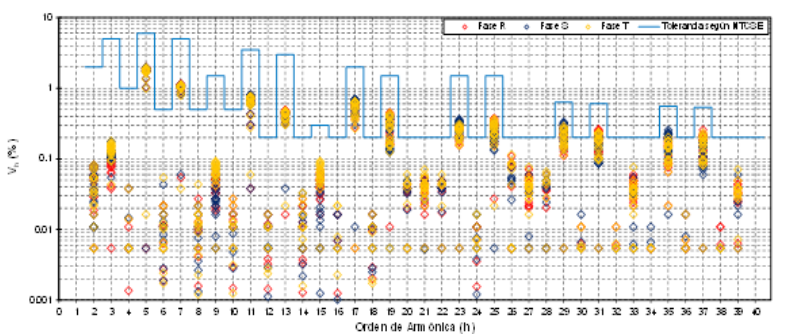

Resultados distorsión armónica total de tensión

Grafico 03. Distorsión armónica total

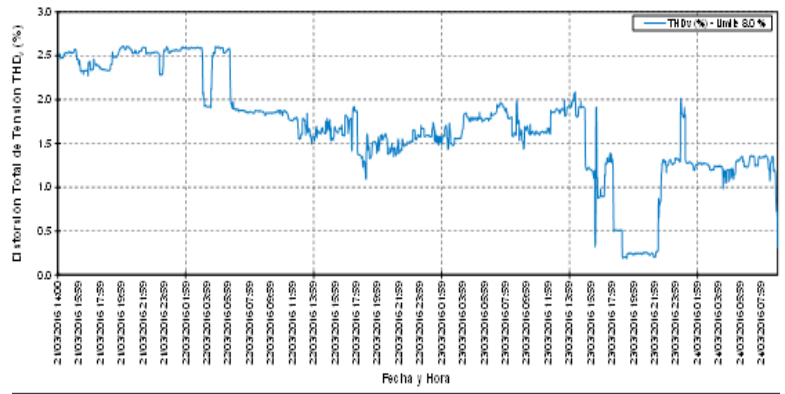

Tabla 04. Distorsión armónica total de tensión THDv

\begin{tabular}{|c|c|c|c|c|c|c|}
\hline \multirow[b]{2}{*}{ ítem } & \multirow[b]{2}{*}{$\begin{array}{l}\text { Punto } \\
\text { de Me- } \\
\text { dición }\end{array}$} & \multirow[b]{2}{*}{$\begin{array}{c}\text { Ten- } \\
\text { sión } \\
\text { No- } \\
\text { minal } \\
(\mathrm{kV})\end{array}$} & \multicolumn{4}{|c|}{ THDV } \\
\hline & & & $\underset{(\%)}{\text { Máximo }}$ & $\begin{array}{c}\text { Promedio } \\
(\%)\end{array}$ & $\underset{(\%)}{\text { Mínimo }}$ & $\begin{array}{c}\text { Toleran- } \\
\text { cia } \\
\text { NTCSE } \\
(\%)\end{array}$ \\
\hline 1 & TD1 & 0.22 & 1.32 & 0.84 & 0.41 & 8.00 \\
\hline
\end{tabular}

En la Tabla 04 se observa que los valores de distorsión armónica de tensión se encuentran dentro de la tolerancia establecida por la NTCSE.

De lo mencionado, se indica que la calidad de energía entregada en el punto es de buena calidad.

Resultado de las armónicas de corriente

Tabla 05. Armónicos de corriente.

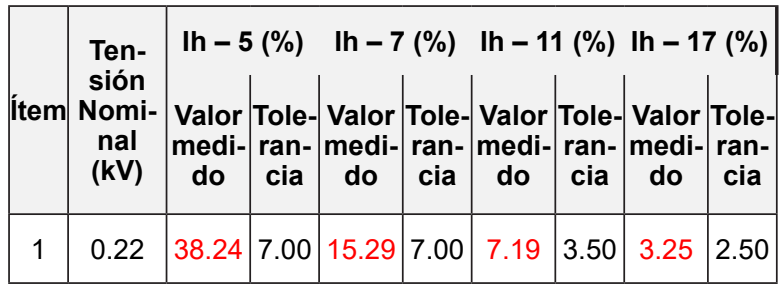

Gráfico 04. Espectro de armónica de corriente

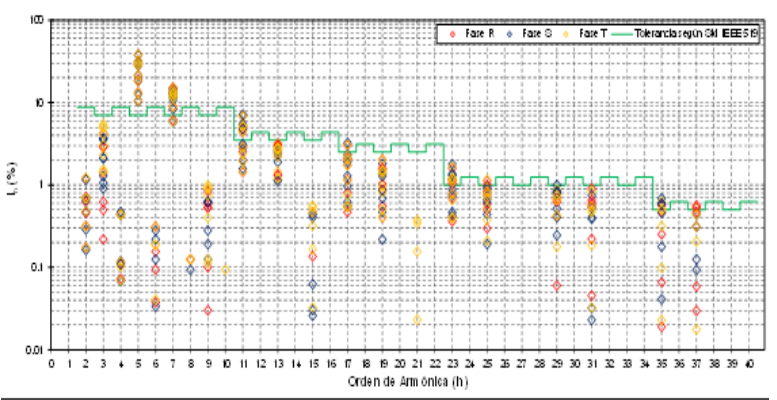

Resultados de la distorsión armónica total de corriente

Tabla 06. Distorsión de la armónica total de corriente THDi.

\begin{tabular}{|c|c|c|c|c|c|c|}
\hline \multirow{2}{*}{ Ítem } & \multirow{2}{*}{$\begin{array}{l}\text { Punto } \\
\text { eléctrico }\end{array}$} & \multicolumn{4}{|c|}{ THDi } & \multirow{2}{*}{$\begin{array}{l}\text { (\%) de } \\
\text { inter- } \\
\text { valos } \\
\text { fuera } \\
\text { de tole- } \\
\text { rancia }\end{array}$} \\
\hline & & Máximo & Promedio & Mínimo & \begin{tabular}{|} 
Tolerancia \\
Std. IEEE \\
519
\end{tabular} & \\
\hline 1 & TD1 & 42.12 & 26.20 & 0.00 & 8.00 & 93.63 \\
\hline
\end{tabular}


Gráfico 05. Comportamiento de la potencia activa y THDi $(\%)$

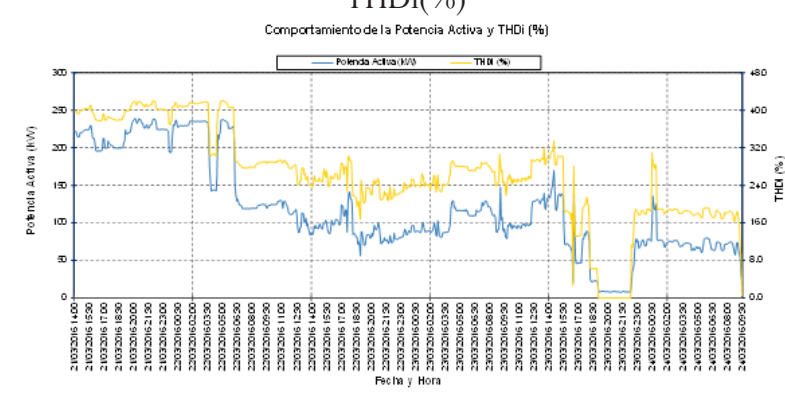

\section{Discusión}

En la gráfica 03 se observa que la tensión RMS no es contante y la variación de tensión fluctúa entre 209 y 231 voltios.

El valor eficaz de la tensión no ha salido del rango $\pm 5 \%$. Si se considerara un tiempo de agregación de $15 \mathrm{~min}$, el porcentaje de tiempo en que la tensión está fuera del rango $\pm 5 \%$ sería obviamente menor. Además, la NTCSE admite que en el $95 \%$ de los casos los valores de la tensión eficaz en 15 minutos no excedan el rango $\pm 5 \%$ alrededor del valor nominal: en base a la norma y a estos resultados se puede concluir que el control de la tensión en la estación transmisora de Radio Universitaria es aceptable.

En la Tabla 03 y Tabla 01, están definidos las tolerancias para cada armónica de acuerdo a la NTCSE, se observa que los valores máximos de armónica de tensión en los puntos de medición se encuentran dentro de las tolerancias establecidas por la NTCSE. Cabe indicar que los valores de las armónicas de tensión mostrados son las más perjudiciales ante un sistema con carga eléctrica.

En la tabla 04 y tabla 01, distorsión armónica total de tensión THDv, se indica el límite máximo de el THDv debe ser menor o igual al $8 \%$. Calculando elTHdv $=5.45 \%$, se observa que el valor de THDv se encuentran dentro de la tolerancia establecida por la NTCSE. De lo mencionado se registra que la calidad de energía entregada en el punto es de buena calidad.

En la Tabla 05, se observa que la armónica de corriente de 5 to orden se encuentra fuera de tolerancia en los puntos de medición, del mismo modo las armónicas de corriente de $7 \mathrm{mo}, 11 \mathrm{vo}$ y $17 \mathrm{vo}$ orden se encuentran fuera de tolerancia en el punto de medición. Comparando con los valores de las tolerancias de la NTCSE indicadas en la tabla 02, se puede ver que la 5 ta armónica es de 38.24 mayor a la tolerancia de 7, la $7 \mathrm{ma}$ armónica es de 15.29 mayor a la tolerancia de 7, la armónica 11 es de 7.19 mayor a la tolerancia de 3.50 y la 17 va armónica de 3.25 supera a la tolerancia de 2.50. Se concluye que el problema que tiene la estación transmisora de Radio Universitaria es con los armónicos de corriente.

De la evaluación de la distorsión armónica total de corriente exceden las tolerancias establecidas en el estándar IEEE 519. Este punto eléctrico posee altos grados de contaminación armónica. Como se indica que es ocasionado por la carga distorsionante instalada en las barras de $0.22 \mathrm{kV}$. (Tabla 06 y Grafico 05).

\section{Conclusiones}

- De la evaluación realizada a las instalaciones de la estación transmisora de Radio Universitaria, la tensión esta dentro de los rangos establecidos por la NTCSE, los armónicos de tensión también están dentro de los rangos establecidos por dicha norma, pero se pueden observar que algunos armónicos y el THDi están fuera de los rangos establecidos por la misma norma. Finalmente se concluye que la calidad de energía de esta estación transmisora no es buena.

- De la evaluación a los valores de tensión RMS, estos se encuentran dentro de la tolerancia establecida por la NTCSE; es decir, el equipamiento se encuentra trabajando dentro de los valores nominales de tensión. De la evaluación a los valores de armónicos de tensión, se indica que estos no superan la tolerancia establecida por la NTCSE; y de la evaluación a los valores de corrientes armónicas, se indica que la presencia de equipamiento electrónico dentro de los equipos de receptores y transmisores originan la inyección de corriente armónica, llegando a sobrepasar las tolerancias recomendadas por el estándar IEEE 519. Se determina que la armónica de 5 to orden produce la variación de tensión y corriente.

- Los armónicos de corriente que se encuentran fuera de la tolerancia permitida por la NTCSE se deben a la falta de filtros en la estación transmisora de Radio Universitaria.

\section{Recomendaciones}

- Si bien los THD de voltaje encontrado tienen valores tolerables, estos voltajes armónicos generan corrientes armónicas en niveles peligrosos para la electrónica de control; por ello, se recomienda instalar un sistema de puesta a tierra adecuado para evitar posibles fallas. Así mismo, que las impedancias de las rutas a tierra tengan valores bajos (menos de $10 \mathrm{Ohm}$ ), y los pozos a tierra deben tener valores de menos de 5 Ohmios.

- Se recomienda instalar un filtro para la 5 ta armónica y $7 \mathrm{ma}$ armónica, para reducir el nivel de variación de la tensión y corriente y, de esta forma, mejorar la calidad de la energía eléctrica. 


\section{Referencias bibliográficas}

IEEE Recommended Practice for Monitoring Electric Power Quality. (1995) (IEEE Std. 1159-1995). Volume: 10. Institute of Electrical and Electronics Engineers. Estados Unidos,

IEEE Recommended Practice and Requirements for Harmonic Control in Electric Power Systems. (1993) (IEEE Std. 519-1992). Institute of Electrical and Electronics Engineers. ISBN 1-55937-239-7. Estados Unidos,.

A. McEachem, W.M. Grady, W.A. Moncrief, G.T. Heydt and M. McGranaghan, (1994) Revenue and Harmonics: An Evaluation of Some Proposed Rate Structures, IEEE Transmission and Distribution. Conference, Transactions paper, April.

La Norma Técnica de Calidad de los Servicios Eléctricos (NTCSE), aprobada por Decreto Supremo No 02097-EM

Cervantes Roa, Óscar Mauricio. (2014) Metodología de Medición de Calidad de Energía Eléctrica en Base a Normas Nacionales e Internacionales para la Universidad De La Costa - CUC, Tesis para obtener el título de Ingeniero Eléctrico. Barranquilla, Colombia.

Gómez Coello, David y Holguín, Marcos, (2010). Análisis de Calidad de Energía Eléctrica en el Nuevo Campus de la Universidad Politécnica Salesiana, Tesis para obtener el título de Ingeniero Eléctrico. Guayaquil, Ecuador.

Ponce De León Cordova, Jean Paul. (2010). Metodología Para la Separación y Cuantificación de las Contribuciones Armónicas en un Punto de Acoplamiento Común (Pac), Tesis para optar el Titulo Profesional de Ingeniero Electricista. UNI. Lima, Peru.

IEEE Recommended Practice and Requirements for Harmonic Control in Electrical Power Systems. (1995). IEEE Std 519-1992pp 23-24. 